and serum HMGB-1 level; whereas, we found a positive correlation with both BDCAF and BSAS ${ }^{2}$. This suggests that HMGB-1 can be used as a new disease activity parameter in BS. In conclusion, this study is unique as it involves the largest number of BS patients and uses BDCAF and BSAS together to assess disease activity

References:

[1] Ahn, J.K., et al., Extracellular high-mobility group box 1 is increased in patients with Behcet's disease with intestinal involvement. Journal of Korean medical science, 2011. 26(5): p. 697-700.

[2] de Souza, A.W., et al., High mobility group box 1 serum levels are increased in Behcet's disease, but not associated with disease activity or disease manifestations. Rheumatology, 2015. 54(12): p. 2151-2155.

Disclosure of Interests: None declared

DOI: 10.1136/annrheumdis-2020-eular.4232

\section{AB0496 $\quad$ AUTOANTIBODIES TARGETING COMPLEMENT RECEPTORS 3A AND 5A1 ARE DECREASED IN ANCA- ASSOCIATED VASCULITIS AND CORRELATE WITH HIGHER RELAPSE RATE.}

S. Klapa ${ }^{1}$, A. Müller ${ }^{2}$, A. Koch ${ }^{3}$, A. Kerstein-Staehle ${ }^{2}$, W. Kaehler ${ }^{3}$, H. Heidecke ${ }^{4}$, S. Schinke ${ }^{2}$, M. Huber-Lang ${ }^{5}$, M. Nitschke ${ }^{6}$, S. Pitann ${ }^{2}$, C. Karsten ${ }^{7}$, G. Riemekasten ${ }^{2}$, P. Lamprecht ${ }^{2}{ }^{1}$ University of Lübeck and Institute of Experimental Medicine, Christian-Albrechts-University of Kiel c/o German Naval Medical Institute, Department of Rheumatology and Clinical Immunology, Lübeck, Germany; ${ }^{2}$ University of Lübeck, Department of Rheumatology and Clinical Immunology, Lübeck, Germany; ${ }^{3}$ Institute of Experimental Immunology, Christian-Albrechts-University Kiel, c/o German Naval Medical Institute, Kronshagen, Germany; ${ }^{4}$ CellTrend $\mathrm{GmbH}$, Luckenwalde, Luckenwalde, Germany; ${ }^{5}$ Institute of Clinical and Experimental Trauma Immunology, University Hospital UIm, UIm, Germany; ${ }^{6}$ Department of Internal Medicine I, Transplant Center, University of Lübeck, Lübeck, Germany; ${ }^{7}$ Institute of Systemic Inflammation Research, University of Lübeck, Lübeck, Germany

Background: Activation of the alternative and final common pathways have been shown in ANCA-associated vasculitis (AAV) (1). Circulating titers of C5a are elevated and correlate with disease activity in AAV. Binding to the corresponding G protein-coupled receptor (GPCR) C5aR1 enhances the influx of neutrophils, leading to ROS generation and severe necrotizing of vascular walls (2). Moreover, subsequent interaction of C5a with C5aR1 may represent a proinflammatory amplification loop (3). Blocking of the receptor is protective in a murine model in AAV (4). In humans, avacopan, a C5aR1-inhibitor showed promising results as glucocorticoid-sparing agent in two randomized phase II and one ongoing phase III clinicals trials in AAV (NCT02994927). Notably, disease-specific anti-GPCR autoantibody (aab) signatures have been found in different autoimmune diseases (5)

Objectives: The aim of the present study was to examine whether (patho)physiological anti-C3aR and anti-C5aR1 aabs correlate with clinical findings in AAV, and whether this is linked to the clinical outcome.

Methods: Sera and plasma of AAV patients [granulomatosis with polyangiitis (GPA), $n=64$; microscopic polyangiitis (MPA), $n=26$; eosinophilic granulomatosis with polyangiitis (EGPA), $n=11$ ] were measured by Elisa for circulating autoantibodies against complement receptors $\mathrm{C} 3 \mathrm{a}$ (anti-C3aR aab) and C5a (anti-C5aR1 aab) and plasma levels of $\mathrm{C} 3 \mathrm{a}$ and $\mathrm{C} 5 \mathrm{a}$. Expression of $\mathrm{C} 3 \mathrm{aR}$ and $\mathrm{C} 5 \mathrm{aR} 1$ on T-cells was determined using flow cytometry. Clinical data were assessed at the time of serum sampling and during follow-up for 48 months Results: GPA displayed low titers of anti-C3aR aab (GPA:5.33 \pm 2.54 vs. $\mathrm{HD}: 6.47 \pm 2.61, \mathrm{P}=0.0031$ ). Anti-C5aR1 aab were decreased in AAV, especially in GPA (GPA: $1.02 \pm 1.07$ vs. HD:6.63 $\pm 2.91, P=<0.0001$ ). Plasma levels of $C 5 a$ and anti-C5aR aab yielded an inverse correlation in AAV ( $r=-0.6813, P=0.0127)$. C5aR1 expression was increased on T-cells in GPA (CD4+C5aR1+T-cells: GPA: $10.76 \pm 2.55 \%$ vs. HD: $3.44 \pm 0.68 \%, \quad \mathrm{P}=0.0021 ; \quad$ CD $8+C 5 a R 1+$ T-cells GPA: $9.74 \pm 2.10 \%$ vs. HD: $4.11 \pm 0.92 \%, P=0.0198)$. Reduced titers of anti-C5aR1 aab $<0.45 \mathrm{U} / \mathrm{ml}$ displayed an increased relapse risk for major organ involvement in GPA (HR 12.85, $\mathrm{P}=0.0014)$.

Conclusion: As potential diagnostic marker, anti-C5aR1 aab titer may additionally be useful to monitor disease activity in AAV.

References:

[1] Chen $\mathrm{M}$ et al. Complement deposition in renal histopathology of patients with ANCA-associated pauci-immune glomerulonephritis. Nephrol Dial Transpl. 2009;24:1247-1252

[2] Schreiber A et al. C5a receptor mediates neutrophil activation an ANCA-induced glomerulonephritis. J Am Soc Nephrol. 2009; 20:289-298

[3] Lamprecht $P$ et al.: Pathogenetic and clinical aspects of Anti-Neutrophil Cytoplasmic Autoantibody-associated vasculitides. Front Immunol. $2018 \mathrm{Apr}$ $9 ; 9-680$
[4] Xiao H et al. C5a receptor (CD88) blockade protects against MPO-ANCA GN. J Am Soc Nephrol. 2014;25(2):225-31

[5] Klapa S et al. Decreased endothelin receptor A autoantibody levels are associated with early ischaemic events in patients with giant-cell arteritis. Ann Rheum Dis 2019 Oct; 78(19):1443-1444

Disclosure of Interests: Sebastian Klapa Grant/research support from: Actelion, Consultant of: Pfizer, Abbvie, Antje Müller: None declared, Andreas Koch None declared, Anja Kerstein-Staehle: None declared, Wataru Kaehler: None declared, Harald Heidecke Shareholder of: Cell Trend GmbH, Employee of: Cell Trend $\mathrm{GmbH}$, Speakers bureau: Cell Trend $\mathrm{GmbH}$, Susanne Schinke Speakers bureau: Pfizer, Markus Huber-Lang: None declared, Martin Nitschke: None declared, Silke Pitann: None declared, Christian Karsten: None declared, Gabriela Riemekasten Consultant of: Cell Trend $\mathrm{GmbH}$, Janssen, Actelion, Boehringer Ingelheim, Speakers bureau: Actelion, Novartis, Janssen, Roche, GlaxoSmithKline, Boehringer Ingelheim, Pfizer, Peter Lamprecht: None declared DOI: 10.1136/annrheumdis-2020-eular.1758

\section{AB0497 RENAL INVOLVEMENT IN ANCA-ASSOCIATED VASCULITIS: DOTHE PRESENCE OF ANCA AND THEIR TYPE MATTER?}

N. Bulanov ${ }^{1}$, E. Stolyarevich ${ }^{2}$, A. Zykova ${ }^{1,3}$, E. Safonova ${ }^{3}$, E. Shchegoleva ${ }^{1}$, E. Kuznezova ${ }^{1}$, M. Bulanova $^{4}$, P. Novikov ${ }^{1}$, S. Moiseev ${ }^{1,3}{ }^{1}$ Sechenov First Moscow State Medical University, Tareev Clinic of Internal Diseases, Moscow, Russian Federation; ${ }^{2}$ A.I. Yevdokimov Moscow State University of Medicine and Dentistry, Moscow, Russian Federation; ${ }^{3}$ Lomonosov Moscow State University, Moscow, Russian Federation; ${ }^{4}$ Vladimir Regional Clinical Hospital, Vladimir, Russian Federation

Background: The role of ANCA type is well established for the risk of relapses of ANCA-associated vasculitis (AAV). However their association with renal involvement and its outcomes is less well understood.

Objectives: To assess clinical and morphological features of ANCA-associated glomerulonephritis (ANCA-GN) and renal survival in ANCA-negative patients, proteinase-3-ANCA (pr3-ANCA) positive and myeloperoxidase-ANCA (MPOANCA) positive patients.

Methods: We enrolled 53 patients with AAV, diagnosed according to Chapel Hill Consensus Conference (2012) definition and/or ACR (1990) criteria, with histologically proven renal involvement. There were $13(24.5 \%)$ males, median age at onset was $48(33 ; 57)$ years. Seven patients were ANCA-negative $(13.3 \%), 17(32.0 \%)$ patients were pr-3-ANCA positive and 29 (54.7\%) patients were MPO-ANCA-positive. ANCA-associates glomerulonephritis (ANCA-GN) class was established according to Berden et al classification. ${ }^{1}$ We retrospectively assessed ANCA rena risk score (ARRS) at disease onset. ${ }^{2}$ Twelve patients $(22.6 \%)$ developed endstage renal disease (ESRD) after a median of $12(6.5 ; 28)$ months. Renal survival rates were assessed by Kaplan-Meier method and compared by log-rank test.

Results: The only significant difference was median BVAS score which was significantly higher in pr3-ANCA-positive $(18(17 ; 20))$ than in MPO-ANCA positive patients $(15(12 ; 18), p=0.012)$. Creatinine levels, eGFR, percentage of glomeruli with crescents, global sclerosis, and interstitial fibrosis and tubular atrophy didn't depend on the presence of ANCA or type of the antibodies The proportion of patients with focal, crescentic, mixed of sclerotic class of ANCA-GN was similar in all groups. There was no significant difference in the numbers of patients with low, medium or high risk of ESRD according to ARRS. One- and three-year renal survival rates were similar in ANCA-negative $(81.7 \%$ and $60.0 \%$ respectively) and ANCA-positive patients $(84.2 \%$ and $74.6 \%$ respectively, Figure $1 \mathrm{~A})$. One-year and three-year survival rates were higher in MPO-ANCA-positive $(84.4 \%$ and $84.4 \%$ respectively) than in pr3-ANCA-positive patients $(73.1 \%$ and $50.1 \%$ respectively), however the difference was not statistically significant (Figure 1B).
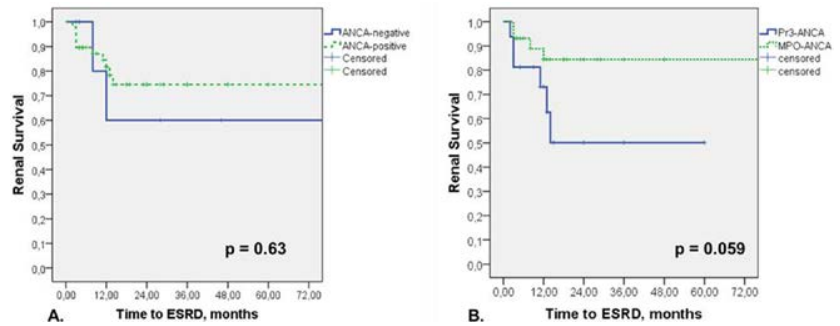

Figure 1. Kaplan-Meier curves showing renal survival in ANCA-positive and ANCA-negative patients (A), and pr3-ANCA-positive and MPO-ANCA-positive patients (B) 
Conclusion: Our small study indicates that clinical and morphological features of renal involvement, as well as renal survival are similar in ANCA-negative and ANCA-positive patients and don't depend on the type of ANCA.

References:

[1] Berden AE, Ferrario F, Hagen EC, et al. Histopathologic Classification of ANCA-Associated Glomerulonephritis. J Am Soc Nephrol. 2010;21(10):1628-1636.

[2] Brix RB, Noriega $M$, Tennstedt $P$, et al. Development and validation of a renal risk score in ANCA-associated glomerulonephritis. Kidney Int. 2018;94(6):1177-1188.

Disclosure of Interests: : Nikolai Bulanov Grant/research support from: This work was supported by the 5-100 Project, Sechenov University, Moscow, Ekaterina Stolyarevich: None declared, Anastasiia Zykova: None declared, Elizaveta Safonova: None declared, Elena Shchegoleva: None declared, Ekaterina Kuznezova: None declared, Mayra Bulanova: None declared, Pavel Novikov Grant/research support from: This work was supported by the 5-100 Project, Sechenov University, Moscow, Sergey Moiseev Grant/research support from: This work was supported by the 5-100 Project, Sechenov University, Moscow

DOI: 10.1136/annrheumdis-2020-eular.6262

\section{AB0498 CHARACTERISTICS AND OUTCOME OF CORONARY ARTERY LESIONS DUE TO POLYARTERITIS NODOSA: ANALYSIS OF A SINGLE CENTER COHORT IN CHINA}

C. Lai ${ }^{1}$, L. Zhao ${ }^{2}$, J. Zhou ${ }^{3}$, D. Xu ${ }^{3}$, X. Tian ${ }^{3}$, X. Zeng ${ }^{3}$, F. Zhang ${ }^{3} .{ }^{1}$ Peking Union Medical College Hospital, Beijing, China; ${ }^{2}$ The Second Hospital of Dalian Medical University, Liaoning, China; ${ }^{3}$ Peking Union Medical College Hospital, Beijing, China

Background: Polyarteritis nodosa (PAN) is a systemic necrotizing vasculitis that mainly affects medium-sized muscular arteries. The coronary artery could be affected. Some severe cases can lead to spontaneous coronary artery dissection (SCAD) and about $0.02 \%$ will die abruptly. Early diagnosis will improve prognosis, but relative studies are all case reports so far.

Objectives: To investigate the clinical characteristics, risk factors and outcome of patients with polyarteritis nodosa (PAN) complicated with coronary artery lesions in China.

Methods: Data of 158 patients with PAN who were admitted to Peking Union Medical College Hospital from September 1986 to September 2019 were retrospective collected. Data were analyzed and compared according to with and without coronary artery lesions due to PAN.

Results: 17 (10.8\%) patients with PAN had the coronary artery lesions due to PAN. The age at coronary artery lesion was $36.9 \pm 10.3$ years. $12(70.6 \%)$ patients were male. There are not statistical differences between two groups in common risk factors of coronary arterial atherosclerosis including smoking, hypertension, diabetes mellitus and hyperlipidemia. Most of them are multi-vessel lesions ( 8 cases are triple-vessel lesions and 3 cases are bi-vessel lesions). Type of coronary artery affected is shown mainly in stenosis (13 cases). Myocardial infarction are shown in 8 cases $(47.1 \%)$. Compared to patients without coronary artery lesions, patients with coronary artery lesions had less nervous system involvement (17.6\% vs.46.8\%) and elevated number of leukocyte (17.6\% vs.56\%). Besides, patients with coronary artery affected exhibit more cranial and carotid artery involvement(29.4\% vs. $5.0 \%)$, renal artery involvement $(41.2 \%$ vs. $17.0 \%)$, coeliac artery involvement (58.8\% vs. $27.0 \%)$, new onset hypertension ( $47.1 \%$ vs. $14.5 \%$ ), renal infarction $(27.3 \%$ vs.5.4\%, ) and higher proportion of 2009 Five-factor score (FFS) $\geq 2$ $(62.5 \%$ vs. $15.6 \%)$. All patients with coronary artery lesions received at least moderate dose of prednisone and CTX except one refused medication. 3 cases underwent interventional therapy. Stent placement was performed on 2 of them, and in-stent restenosis was appeared in a patient one year later. 2 cases died,one for vascular rupture after coronary aneurysmsutrue plus coronary artery bypass grafting, another for myocardial infarction after stopping immunosuppressant therapy himself.Survival analysis showed patients with digital $\mathrm{g}$ angrene had poor prognosis though no significant difference $(p=0.055)$

Conclusion: PAN with coronary artery lessions are not uncommon. These patients exhibit young age, more proportion of multi-vessel of coronary artery involvement, more combined involvments of other organ arteries and more severe disease.

\section{References:}

[1] Munguti CM, Ndunda PM, Muutu TM. Sudden Death From Spontaneous Coronary Artery Dissection Due to Polyarteritis Nodosa. Cureus, 2017;9 (10), e1737

[2] Kritta nawong C, Kumar A, Johnson KW, et al.Conditions and Factors Associated With Spontaneous Coronary Artery Dissection (From a National Population-Based Cohort Study).Am J Cardiol, 2019; 123 (2): 249-253

[3] Hwang J, Yang JH, Kim DK, Cha HS.Polyarteritis Nodosa Involving Rena and Coronary Arteries. J Am Coll Cardiol. 2012;59(7):e13

Disclosure of Interests: : Chinchih Lai: None declared, Lin Zhao: None declared, Jiaxin Zhou: None declared, Dong Xu: None declared, Xinping Tian: None declared, Xiaofeng Zeng Consultant of: MSD Pharmaceuticals, Fengchun Zhang: None declared

DOI: 10.1136/annrheumdis-2020-eular.4343

\section{AB0499 $\quad$ ABNORMALITY OF PERIPHERAL LYMPHOCYTE SUBSETS IN BECHET'S DISEASE AND EFFECTS OF NEW IMMUNOREGULATORY COMBINATION THERAPIES ON THESE CELLS}

Q. Y. Su ${ }^{1}$, S. X. Zhang ${ }^{2}$, L. M. Hao ${ }^{2}$, J.Y. Yang ${ }^{1}$, J. Bai ${ }^{1}$, S. J. Guo ${ }^{1}$, J. Luo ${ }^{2}$, G. Y. Liu' ${ }^{2}, \mathrm{C} . \mathrm{Gao}^{3}, \underline{\mathrm{X} . \mathrm{LI}^{2}} .{ }^{1}$ Shanxi Medical University, Taiyuan, China; ${ }^{2}$ the Second Hospital of Shanxi Medical University, Taiyuan, China; ${ }^{3}$ Brigham and Women's Hospital, Harvard Medical School, Boston, United States of America

Background: Bechet's disease (BD) is a chronic multisystemic vasculitis Although its exact etiopathology is unknown, both autoimmune imbalances associated with genetic and abnormal immune response of effector lymphocytes promoted by infectious factors are suggested ${ }^{1}$. The increase of effector $T$ cells (Teffs) and the decrease of regulatory $T$ cells (Tregs) are possibly the involving factors in the pathogenesis of $\mathrm{BD}^{2}$. Importantly, we have developed new immunoregulatory combination therapies trying to restore the reduction of Tregs in rheumatic patients.

Objectives: To examine abnormal levels of lymphocyte subsets in BD patients at a relatively large-sample size and to investigate whether the immunoregulatory combination therapies have therapeutic efficacy in BD.

Methods: Total 384 BD patients and 206 healthy controls (HCs) were enrolled in this cross-sectional study. Proportions and absolute numbers of peripheral T, $\mathrm{B}, \mathrm{NK}, \mathrm{CD}^{+} \mathrm{T}, \mathrm{CD}^{+} \mathrm{T}, \mathrm{Th} 1, \mathrm{Th} 2, \mathrm{Th} 17$ and Treg subsets were analyzed by flow cytometry (FCM) for all participants. Among these patients, 183 cases of BD patients were treated with immunoregulatory combination drugs (IMiDs) such as low-dose interleukin-2, rapamycin, metformin, retinoic acid and coenzyme Q10. The levels of peripheral lymphocyte subsets were measured before and after the treatment. Compared-T test was used to compare continuous measures and to assess effect of these drugs.

Results: Compared to HCs, the absolute numbers of various Teffs such as T, $\mathrm{B}, \mathrm{CD} 4^{+} \mathrm{T}, \mathrm{CD} 8^{+} \mathrm{T}$, Th1 and Th17 cells were significantly increased in BD group $(P<0.01)$, while the level of Tregs in patients with BD was severely decreased $(P<0.05)$, resulting in increased ratios (imbalance) of Th1/Tregs, Th2/Tregs and Th17/Tregs $(P<0.05)$ (Figure 1). After the IMiDs treatment, the levels of NK, $\mathrm{CD}^{+} \mathrm{T}, \mathrm{CD}^{+} \mathrm{T}$, Th1, Th17 cells as well as Tregs were significantly increased $(P<0.05)$. But the increased Tregs was much more dramatical than those of Teffs, resulting in a decrease in ratios of Teffs/Tregs such as Th2/Tregs $(P<$ 0.001) (Figure 2).

Conclusion: Impaired balance of pro- and anti-inflammatory immune cells, especially insufficiency of Tregs, might be a cornerstone of the pathogenesis of BD. Immunoregulatory combination therapies could promote the proliferation and functional recovery of Tregs in patients with BD and might help to alleviate disease activity.

References:

[1] Yazici H, Seyahi E, Hatemi G, et al. Behcet syndrome: a contemporary view. Nat Rev Rheumatol 2018;14(2):107-19. doi: 10.1038/nrrheum.2017.208 [published Online First: 2018/01/04]

[2] Rosenzwajg M, Lorenzon R, Cacoub P, et al. Immunological and clinical effects of low-dose interleukin-2 across 11 autoimmune diseases in a single, open clinical trial. Ann Rheum Dis 2019;78(2):209-17. doi: 10.1136/annrheumdis-2018-214229 [published Online First: 2018/11/26] 